\title{
NOT gate response in a mesoscopic ring: An exact result
}

\author{
Santanu K. Maiti ${ }^{1,2, *}$ \\ ${ }^{1}$ Theoretical Condensed Matter Physics Division, Saha Institute of Nuclear Physics, \\ 1/AF, Bidhannagar, Kolkata-700 064, India \\ ${ }^{2}$ Department of Physics, Narasinha Dutt College, 129, Belilious Road, Howrah-711 101, India
}

\begin{abstract}
We explore NOT gate response in a mesoscopic ring threaded by a magnetic flux $\phi$. The ring is attached symmetrically to two semi-infinite one-dimensional metallic electrodes and a gate voltage, viz, $V_{a}$, is applied in one arm of the ring which is treated as the input of the NOT gate. The calculations are based on the tight-binding model and the Green's function method, which numerically compute the conductance-energy and current-voltage characteristics as functions of the ring-to-electrodes coupling strength, magnetic flux and gate voltage. Our theoretical study shows that, for $\phi=\phi_{0} / 2\left(\phi_{0}=c h / e\right.$, the elementary flux-quantum) a high output current (1) (in the logical sense) appears if the input to the gate is low (0), while a low output current (0) appears when the input to the gate is high (1). It clearly exhibits the NOT gate behavior and this aspect may be utilized in designing an electronic logic gate.
\end{abstract}

PACS No.: 73.23.-b; 73.63.Rt.

Keywords: Mesoscopic ring; Conductance; $I-V$ characteristic; NOT gate.

*Corresponding Author: Santanu K. Maiti

Electronic mail: santanu.maiti@saha.ac.in 


\section{Introduction}

The study of electron transport in quantum confined geometries has become one of the most fascinating branch of nanoscience and technology. With the help of different advanced technologies, the simple looking quantum confined systems can be used in designing nanodevices especially in electronic as well as spintronic engineering. The idea of manufacturing nanodevices are based on the concept of quantum interference effect which is generally vanishes for larger systems. On the other hand, for much smaller sizes the quantum phase coherence is maintained across the sample. A mesoscopic metallic ring is one such promising example where electronic motion is confined and the transport becomes predominantly coherent. Using a mesoscopic ring we can make a device that can act as a logic gate, which may be used in nanoelectronic circuits. To explore this phenomenon we design a bridge system where the ring is sandwiched between two external electrodes, so-called the electrode-ring-electrode bridge. The ring is then subjected to an Aharonov-Bohm (AB) flux $\phi$ which is the key controlling factor for the whole logical operation in this particular geometry. The theoretical description of electron transport in a bridge system has got much progress following the pioneering work of Aviram and Ratner. ${ }^{1}$ Later, many excellent experiments ${ }^{2-4}$ have been done in several bridge systems to understand the basic mechanisms underlying the electron transport. Though extensive studies on electron transport have already been done both theoretically ${ }^{5-13}$ as well as experimentally, ${ }^{2-4}$ yet lot of controversies are still present between the theory and experiment, and the complete knowledge of the conduction mechanism in this scale is not very well established even today. For illustrative purposes, here we mention some of these issues as follow. The electronic transport in the ring changes drastically depending on the interface geometry between the ring and the electrodes. By changing the geometry, one can tune the transmission probability of an electron across the ring which is solely due to the effect of quantum interference among the electronic waves passing through different arms of the ring. Not only that, the electron transport in the ring can be modulated in other way by tuning the magnetic flux, that threads the ring. The $\mathrm{AB}$ flux threading the ring may change the phases of the wave functions propagating along the different arms of the ring leading to constructive or destructive interferences, and therefore, the transmission amplitude changes. ${ }^{14-18}$ Beside these factors, ring-to-electrodes coupling is another important issue that controls the electron transport in a meaningful way. ${ }^{18}$ All these are the key factors which regulate the electron transmission in the electrode-ring-electrode bridge system and these effects have to be taken into account properly to reveal the transport mechanisms.

Our main aim of the present work is to study the NOT gate response in a mesoscopic ring threaded by a magnetic flux $\phi$. The ring is contacted symmetrically to the electrodes, and a gate voltage $V_{a}$ is applied in one arm of the ring (see Fig. 1) which is regarded as the input of the NOT gate. A simple tight-binding model is used to describe the system and all the calculations are done numerically. Here we address the NOT gate behavior by studying the conductance-energy and current-voltage characteristics as functions of the ring-electrodes coupling strength, magnetic flux and gate voltage. Our study reveals that for a particular value of the magnetic flux, $\phi=\phi_{0} / 2$, a high output current (1) (in the logical sense) is available if the input to the gate is low (0), while if the input to the gate is high (1), a low output current (0) appears. This phenomenon clearly shows the NOT gate behavior. To the best of our knowledge the NOT gate response in such a simple system has yet not been addressed in the literature.

The scheme of the paper is as follow. Following the introduction (Section 1), in Section 2, we describe the model and the theoretical formulations for the calculation. Section 3 explores the results, and finally, we conclude our study in Section 4.

\section{Model and the synopsis of the theoretical background}

Let us start with the model presented in Fig. 1. A mesoscopic ring, subjected to an $\mathrm{AB}$ flux $\phi$, is attached symmetrically (upper and lower arms have equal number of lattice points) to two semi-infinite one-dimensional (1D) metallic electrodes. A gate voltage $V_{a}$, taken as the input voltage of the NOT gate, is applied to the atomic site $a$ in the upper arm of the ring. While, an additional gate voltage $V_{\alpha}$ is applied to the site $\alpha$ in the lower arm of the ring. Both these two voltages are variable.

At very low temperature and bias voltage the conductance $g$ of the ring can be expressed from 
the Landauer conductance formula, ${ }^{19-20}$

$$
g=\frac{2 e^{2}}{h} T
$$

where $T$ gives the transmission probability of an electron across the ring. This $(T)$ can be represented in terms of the Green's function of the ring and its coupling to the two electrodes by the relation, ${ }^{19-20}$

$$
T=\operatorname{Tr}\left[\Gamma_{S} G_{R}^{r} \Gamma_{D} G_{R}^{a}\right]
$$

where $G_{R}^{r}$ and $G_{R}^{a}$ are respectively the retarded and advanced Green's functions of the ring including the

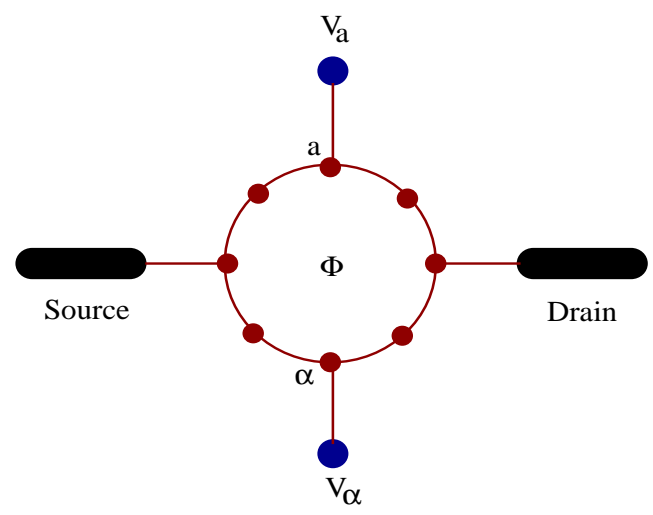

Figure 1: Schematic representation for the operation of a NOT gate. The atomic sites $a$ and $\alpha$ are subjected to the voltages $V_{a}$ and $V_{\alpha}$ respectively, those are variable (for color illustration, see the web version).

effects of the electrodes. Here $\Gamma_{S}$ and $\Gamma_{D}$ describe the coupling of the ring to the source and drain respectively. For the complete system i.e., the ring, source and drain, the Green's function is defined as,

$$
G=(E-H)^{-1}
$$

where $E$ is the injecting energy of the source electron. To Evaluate this Green's function, the inversion of an infinite matrix is needed since the complete system consists of the finite ring and the two semi-infinite electrodes. However, the entire system can be partitioned into sub-matrices corresponding to the individual sub-systems and the Green's function for the ring can be effectively written as,

$$
G_{R}=\left(E-H_{R}-\Sigma_{S}-\Sigma_{D}\right)^{-1}
$$

where $H_{R}$ is the Hamiltonian of the ring that can be expressed within the non-interacting picture like,

$$
H_{R}=\sum_{i}\left(\epsilon_{i 0}+V_{a} \delta_{i a}+V_{\alpha} \delta_{i \alpha}\right) c_{i}^{\dagger} c_{i}
$$

$$
+\sum_{<i j>} t\left(c_{i}^{\dagger} c_{j} e^{i \theta}+c_{j}^{\dagger} c_{i} e^{-i \theta}\right)
$$

In this Hamiltonian $\epsilon_{i 0}$ 's are the site energies for all the sites $i$ except the sites $i=a$ and $\alpha$ where the gate voltages $V_{a}$ and $V_{\alpha}$ are applied, those are variable. These gate voltages can be incorporated through the site energies as expressed in the above Hamiltonian. $c_{i}^{\dagger}\left(c_{i}\right)$ is the creation (annihilation) operator of an electron at the site $i$ and $t$ is the nearest-neighbor hopping integral. The phase factor $\theta=2 \pi \phi / N \phi_{0}$ comes due to the flux $\phi$ threaded by the ring, where $N$ corresponds to the total number of atomic sites in the ring. Similar kind of tightbinding Hamiltonian is also used, except the phase factor $\theta$, to describe the $1 \mathrm{D}$ perfect electrodes where the Hamiltonian is parametrized by constant on-site potential $\epsilon_{0}$ and nearest-neighbor hopping integral $t_{0}$. The hopping integral between the source and the ring is $\tau_{S}$, while it is $\tau_{D}$ between the ring and the drain. The parameters $\Sigma_{S}$ and $\Sigma_{D}$ in Eq. (4) represent the self-energies due to the coupling of the ring to the source and drain respectively, where all the informations of this coupling are included into these self-energies.

The current passing through the ring is depicted as a single-electron scattering process between the two reservoirs of charge carriers. The current $I$ can be computed as a function of the applied bias voltage $V$ by the expression, ${ }^{19}$

$$
I(V)=\frac{e}{\pi \hbar} \int_{E_{F}-e V / 2}^{E_{F}+e V / 2} T(E) d E
$$

where $E_{F}$ is the equilibrium Fermi energy. Here we assume that the entire voltage is dropped across the ring-electrode interfaces, and it is examined that under such an assumption the $I$ - $V$ characteristics do not change their qualitative features.

All the results in this communication are determined at absolute zero temperature, but they should valid even for finite temperature $(\sim 300 \mathrm{~K})$, since the broadening of the energy levels of the ring due to its coupling with the electrodes becomes much larger than that of the thermal broadening. ${ }^{19}$ For simplicity, we take the unit $c=e=h=1$ in our present calculation.

\section{Results and discussion}

To discuss the results, first we mention the values of the different parameters those are used for the 
numerical calculation. The on-site energy $\epsilon_{i 0}$ of the ring is taken as 0 for all the sites $i$, except the sites $i=a$ and $\alpha$ where the site energies are taken as $V_{a}$ and $V_{\alpha}$ respectively, and the nearest-neighbor hopping strength $t$ is set to 3 . On the other hand, for the side attached electrodes the on-site energy $\left(\epsilon_{0}\right)$ and the nearest-neighbor hopping strength $\left(t_{0}\right)$ are fixed to 0 and 4 respectively. The voltage $V_{\alpha}$ is set to 2 . Throughout the study, we focus our results
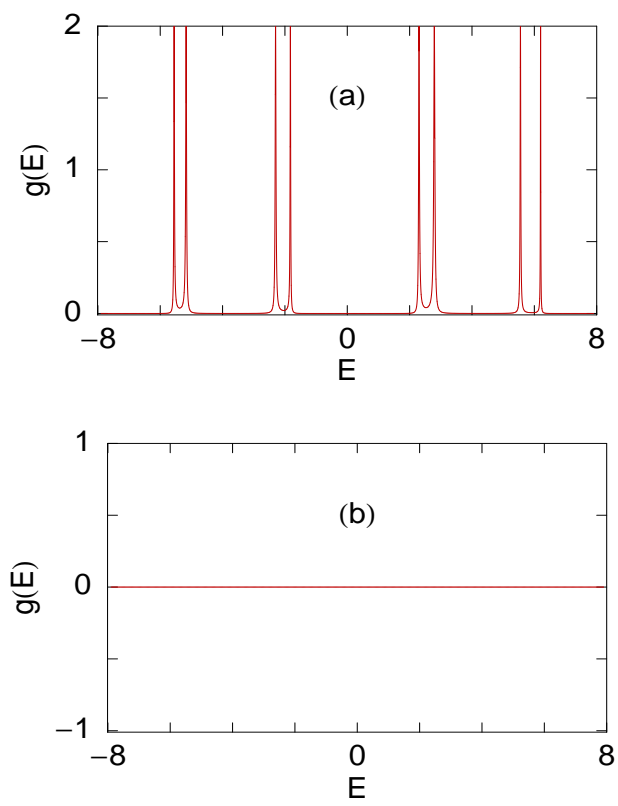

Figure 2: $g$ - $E$ curves in the weak-coupling limit for a mesoscopic ring with $N=8, V_{\alpha}=2$ and $\phi=0.5$. (a) $V_{a}=0$ and (b) $V_{a}=2$ (for color illustration, see the web version).

for the two limiting cases depending on the strength of the coupling of the ring to the source and drain. In one case we use the condition $\tau_{S(D)}<<t$, which is so-called the weak-coupling limit. For this regime we choose $\tau_{S}=\tau_{D}=0.5$. In the other case the condition $\tau_{S(D)} \sim t$ is used, which is named as the strong-coupling limit. In this particular regime, the values of the parameters are set as $\tau_{S}=\tau_{D}=2.5$. The significant parameter for all these calculations is the magnetic flux $\phi$ which is set to $\phi_{0} / 2$ i.e., 0.5 in our chosen unit.

In Fig. 2 2 we show the variation of the conductance $(g)$ as a function of the injecting electron energy $(E)$, in the limit of weak-coupling, for a mesoscopic ring with $N=8$ and $V_{\alpha}=2$. Figures 2(a) and (b) correspond to the results for the input voltages $V_{a}=0$ and $V_{a}=2$ respectively. For the particular case when the input voltage $V_{a}=2$ i.e., the input is high, the conductance $g$ vanishes (Fig. 2(b)) in the complete energy range. This indicates that the conduction of the electron from the source to drain through the ring is not possible. The situation becomes completely different for the case when the input to the gate is zero $\left(V_{a}=0\right)$. The result is shown in Fig. 2(a), where the conductance shows sharp resonance peaks for some fixed energies. This
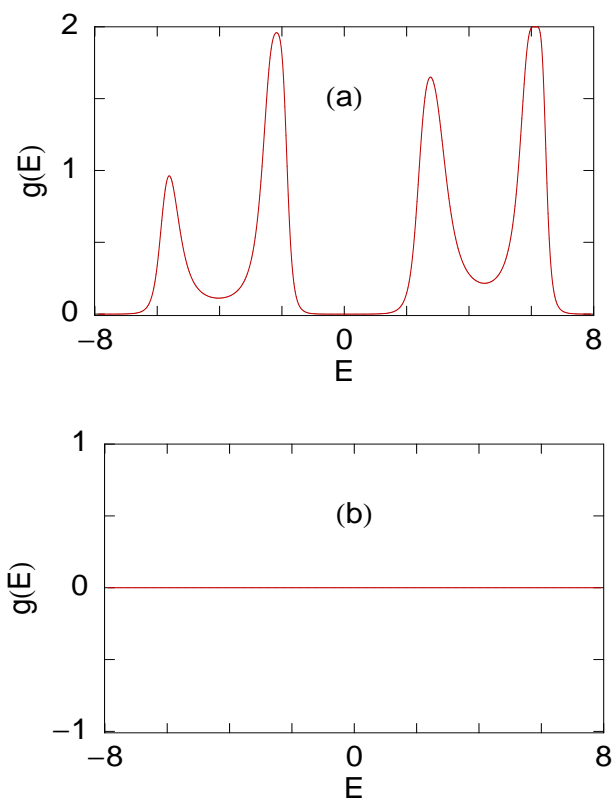

Figure 3: $g-E$ curves in the strong-coupling limit for a mesoscopic ring with $N=8, V_{\alpha}=2$ and $\phi=0.5$. (a) $V_{a}=0$ and (b) $V_{a}=2$ (for color illustration, see the web version).

reveals the electron conduction across the ring. At these resonance $g$ approaches the value 2 , and therefore, the transmission probability $T$ becomes unity, since the expression $g=2 T$ is satisfied from the Landauer conductance formula (see Eq. (1) with $e=h=1$ ). These resonance peaks are associated with the energy eigenvalues of the ring, and thus, it can be predicted that the conductance spectrum manifests itself the electronic structure of the ring. Hence, more resonance peaks are expected for the larger rings, associated with their energy spectra. Now we focus the dependences of the gate voltages on the electron transport for the two different cases of the input voltage. The transmission probability of getting an electron through the ring depends on the quantum interference of the electronic waves passing through the two arms (upper and lower) 
of the ring. For the symmetrically connected ring i.e., when the two arms of the ring are identical with each other, the probability amplitude is exactly zero $(T=0)$ for the flux $\phi=\phi_{0} / 2$. This is due to the result of the quantum interference among the two waves in the two arms of the ring, which can be established by a very simple mathematical calculation. Therefore, for the case when the input to the gate is equal to 2 i.e., $V_{a}=2$, the upper and lower arms of the ring become exactly similar. This is because the potential $V_{\alpha}$ is also set to 2. Accordingly, the transmission probability drops to zero. If the input voltage $V_{a}$ is different from the potential applied in the atomic site $\alpha$, then the
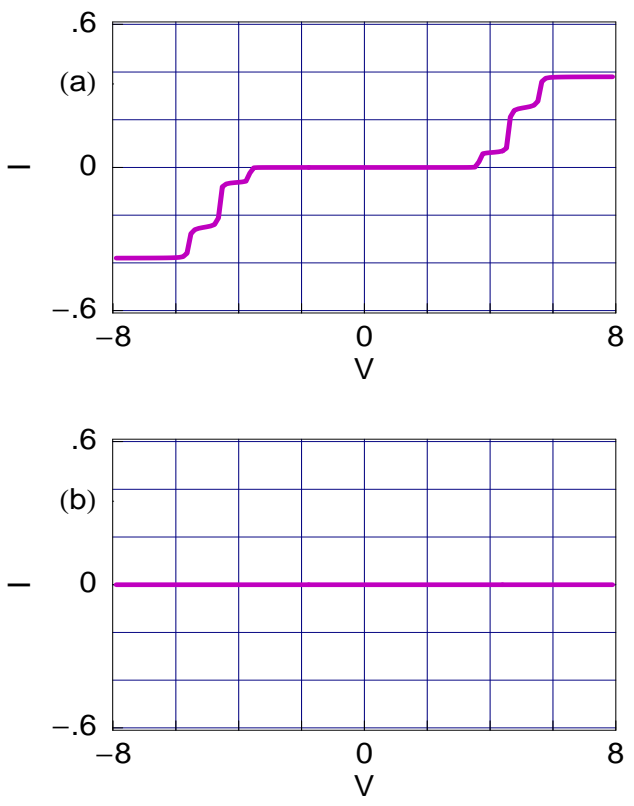

Figure 4: $I$ - $V$ curves in the weak-coupling limit for a mesoscopic ring with $N=8, V_{\alpha}=2$ and $\phi=0.5$. (a) $V_{a}=0$ and (b) $V_{a}=2$ (for color illustration, see the web version).

two arms are not identical with each other and the transmission probability will not vanish. Thus, to get the zero transmission probability when the input is high, we should tune $V_{\alpha}$ properly, observing the input potential and vice versa. On the other hand, due to the breaking of the symmetry of the two arms, the non-zero value of the transmission probability is achieved in the particular case where the input voltage is zero $\left(V_{a}=0\right)$, which reveals the electron conduction across the ring. From these results we can emphasize that the electron conduction takes place through the ring if the input to the gate is zero, while if the input is high, the conduction is no longer possible. This aspect clearly describe the NOT gate behavior. Following with this, here we also describe the effect of the ring-to-electrodes coupling. As representative examples, in Fig. 3 we plot the conductance-energy characteristics for the strong-coupling limit, where (a) and (b) are drawn respectively for the same input voltages as in Fig. 2 . In the strong-coupling case, all the resonances get substantial widths compared to the weak-coupling case. This is due to the broadening of the energy levels of the ring in the limit of strong coupling, where the contribution comes from the imaginary parts of the self-energies $\Sigma_{S}$ and $\Sigma_{D}$ respectively. ${ }^{19}$ Therefore, by tuning the coupling strength, we can get the electron transmission across the ring for the wider range of energies and it provides an important behavior in the study of current-voltage $(I-V)$ characteristics.

All these features of electron transport become much more clearly illustrated from our presented current-voltage $(I-V)$ characteristics. The current

Table 1: NOT gate behavior in the limit of weakcoupling. The current $I$ is computed at the bias voltage 6.02 .

\begin{tabular}{|c|c|}
\hline \hline Input $\left(V_{a}\right)$ & Current $(I)$ \\
\hline 0 & 0.378 \\
\hline 2 & 0 \\
\hline \hline
\end{tabular}

across the ring is determined by integrating the transmission function $T$ as prescribed in Eq. (6). The transmission function varies exactly similar to that of the conductance spectrum, differ only in magnitude by the factor 2 since the relation $g=$ $2 T$ holds from the Landauer conductance formula Eq. (11). As illustrative examples, in Fig. [1 we plot the $I-V$ characteristics for a mesoscopic ring with $N=8$ and $V_{\alpha}=2$, in the limit of weak-coupling.

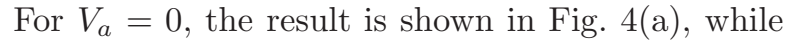
Fig. 4(b) gives the result for $V_{a}=2$. Clearly we see that, when the input to the gate is identical to 2 (high), the current $I$ becomes exactly zero (see Fig. (4)(b)) for the entire bias voltage $V$. This feature is understood from the conductance spectrum, Fig. 2(b), since the current is computed from the integration method of the transmission function $T$. On the other hand, a non-zero value of the current is obtained when the input voltage $V_{a}=0$, as given in Fig. 4 (a). The figure shows that the current exhibits staircase-like structure with fine steps as a function 
of the applied bias voltage. This is due to the existence of the fine resonance peaks in the conductance spectrum in the weak-coupling limit. With the increase of the bias voltage $V$, the electrochemical potentials on the electrodes are shifted gradually, and finally cross one of the quantized energy levels of
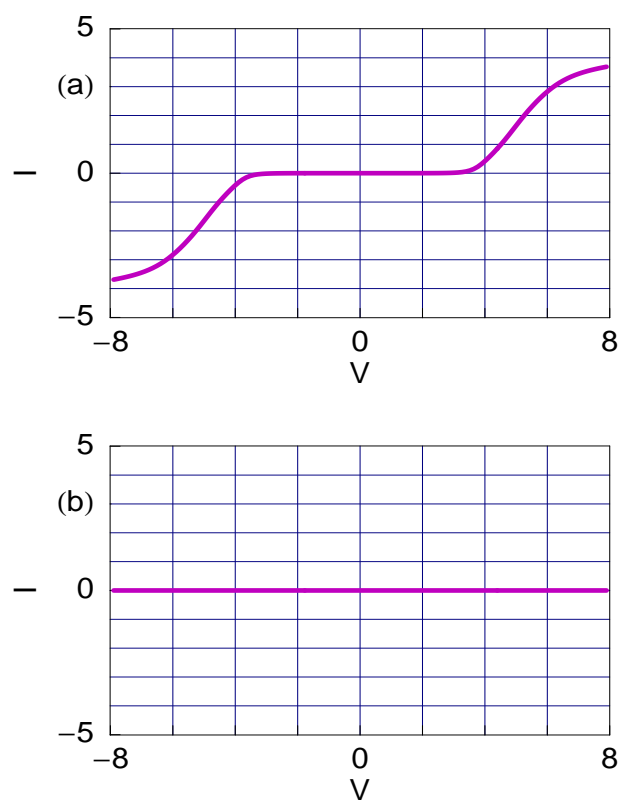

Figure 5: $I$ - $V$ curves in the strong-coupling limit for a mesoscopic ring with $N=8, V_{\alpha}=2$ and $\phi=0.5$. (a) $V_{a}=0$ and (b) $V_{a}=2$ (for color illustration, see the web version).

the ring. Accordingly, a current channel is opened up which provides a jump in the $I-V$ characteristic curve. Here, it is also important to note that the non-zero value of the current appears beyond a finite value of the bias voltage $V$, so-called the

Table 2: NOT gate behavior in the limit of strongcoupling. The current $I$ is computed at the bias voltage 6.02 .

\begin{tabular}{|c|c|}
\hline \hline Input $\left(V_{a}\right)$ & Current $(I)$ \\
\hline 0 & 2.846 \\
\hline 2 & 0 \\
\hline \hline
\end{tabular}

threshold voltage $\left(V_{t h}\right)$. This $V_{t h}$ can be changed by controlling the size $(N)$ of the ring. From these current-voltage characteristic curves, the NOT gate behavior of the ring can be observed very nicely. To make it much clear, in Table 1, we show a quantitative estimate of the typical current amplitude determined at the bias voltage $V=6.02$. It shows that, when the input to the gate is zero, the current gets the value 0.378 . While, the current becomes exactly zero when the input voltage $V_{a}=2$. In the same fashion, as above, here we also describe the $I-V$ characteristics for the limit of strong-coupling. In this limit, the current varies almost continuously with the applied bias voltage and gets much larger amplitude than the weak-coupling case as presented in Fig. 5 The fact is that, in the limit of strongcoupling all the energy levels get broadened which provide larger current in the integration procedure of the transmission function $T$. Thus by tuning the strength of the ring-to-electrodes coupling, we can achieve very large current amplitude from the very low one for the same bias voltage $V$. All the other properties i.e., the dependences of the gate voltages on the $I-V$ characteristics are exactly similar

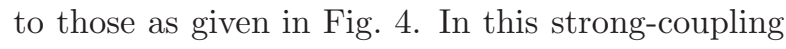
limit we also make a quantitative study for the typical current amplitude, given in Table 2 where the current amplitude is determined at the same bias voltage $(V=6.02)$ as earlier. The response of the output current is exactly similar to that as given in Table 1. Here the current achieves the value 2.846 when the input to the gate is zero $\left(V_{a}=0\right)$, while it $(I)$ becomes exactly zero for the case where $V_{a}=2$. The non-zero value of the current in this strong-coupling limit is much larger than the weakcoupling case, as expected. From these results we can clearly manifest that a mesoscopic ring exhibits the NOT gate response.

\section{Concluding remarks}

In this presentation, we have addressed the NOT gate response in a mesoscopic metallic ring threaded with a magnetic flux $\phi$ in the Green's function formalism. The ring is attached symmetrically to the electrodes and a gate voltages $V_{a}$ is applied in one arm of the ring which is taken as the input of the NOT gate. A simple tight-binding model is used to describe the system and all the calculations are done numerically. We have computed the conductanceenergy and current-voltage characteristics as functions of the gate voltage, ring-to-electrodes coupling strength and magnetic flux. Very interestingly we have observed that, for the half flux-quantum value of $\phi\left(\phi=\phi_{0} / 2\right)$, a high output current (1) (in the logical sense) appears if the input to the gate is low (0). While, if the input to the gate is high (1), a low 
output current (0) appears. It clearly manifests the NOT gate behavior and this aspect may be utilized in designing a tailor made electronic logic gate.

The importance of this article is particularly concerned with (i) the simplicity of the geometry and (ii) the smallness of the size. To the best of our knowledge the NOT gate response in such a simple low-dimensional system that can be operated even at finite temperature $(\sim 300 \mathrm{~K})$ has not been addressed earlier in the literature.

\section{References}

[1] A. Aviram and M. Ratner, Chem. Phys. Lett. 29, 277 (1974).

[2] J. Chen, M. A. Reed, A. M. Rawlett, and J. M. Tour, Science 286, 1550 (1999).

[3] M. A. Reed, C. Zhou, C. J. Muller, T. P. Burgin, and J. M. Tour, Science 278, 252 (1997).

[4] T. Dadosh, Y. Gordin, R. Krahne, I. Khivrich, D. Mahalu, V. Frydman, J. Sperling, A. Yacoby, and I. Bar-Joseph, Nature 436, 677 (2005).

[5] P. A. Orellana, M. L. Ladron de Guevara, M. Pacheco, and A. Latge, Phys. Rev. B 68, 195321 (2003).

[6] P. A. Orellana, F. Dominguez-Adame, I. Gomez, and M. L. Ladron de Guevara, Phys. Rev. B 67, 085321 (2003).

[7] A. Nitzan, Annu. Rev. Phys. Chem. 52, 681 (2001).

[8] A. Nitzan and M. A. Ratner, Science 300, 1384 (2003).

[9] V. Mujica, M. Kemp, and M. A. Ratner, J. Chem. Phys. 101, 6849 (1994).

[10] V. Mujica, M. Kemp, A. E. Roitberg, and M. A. Ratner, J. Chem. Phys. 104, 7296 (1996).

[11] K. Walczak, Phys. Stat. Sol. (b) 241, 2555 (2004).

[12] K. Walczak, arXiv:0309666.

[13] W. Y. Cui, S. Z. Wu, G. Jin, X. Zhao, and Y. Q. Ma, Eur. Phys. J. B. 59, 47 (2007).

[14] R. Baer and D. Neuhauser, J. Am. Chem. Soc. 124, 4200 (2002).
[15] D. Walter, D. Neuhauser, and R. Baer, Chem. Phys. 299, 139 (2004).

[16] K. Tagami, L. Wang, and M. Tsukada, Nano Lett. 4, 209 (2004).

[17] K. Walczak, Cent. Eur. J. Chem. 2, 524 (2004).

[18] R. Baer and D. Neuhauser, Chem. Phys. 281, 353 (2002).

[19] S. Datta, Electronic transport in mesoscopic systems, Cambridge University Press, Cambridge (1997).

[20] M. B. Nardelli, Phys. Rev. B 60, 7828 (1999). 